\title{
The Stress and Stiffness Analysis of Diaphragm
}

\author{
Dongyue Qu , Xiuming Li, Fanbing Liu and Chunhua Zhao \\ School of Harbin Engineering University, Harbin 150000, China
}

\begin{abstract}
Diaphragm coupling with its simple structure, small size, high reliability, which can compensate for its input and output displacement deviation by its elastic deformation, is widely used in aerospace, marine, and chemical etc. This paper uses the ANSYS software and its APDL language to analysis the stress distribution when the diaphragm under the load of torque, axial deviation, centrifugal force, angular deviation and multiple loads. We find that the value of maximum stress usually appears in the outer or inner transition region and the axial deviation has a greater influence to the distribution of the stress. Based on above, we got three kinds of stiffness for axial, angular and torque, which the stiffness of diaphragm is nearly invariable. The results can be regard as an important reference for design and optimization of diaphragm coupling.
\end{abstract}

\section{Introduction}

Diaphragm coupling belongs to metal flexible coupling (fig 1), which is made up of left-right diaphragm, inputoutput shaft and middle shaft, not only can transfer big torque but also deviate the axial, angular and radial displacement by its elastic deformation of profile.

The working principles of the Diaphragm coupling are described below: the torque is inputted from the input shaft by the circumferentially spaced strength bolts. Then, the torque is transmitted to the middle term of the Diaphragm coupling. Besides, the torque is outputted by the same method at another side, which like the input shaft. What's more, diaphragm is a significant component of the metal flexible coupling. When the coupling works, the profile of diaphragm not only transmits the torque between the input and output but also compensate the displacement deviation by its elastic deformation [1], etc. Usually, the diaphragm coupling suffers centrifugal force, torque, and axial deformation loads. The design of profile plays an important role in design of the diaphragm coupling. From that, by taking the conical diaphragm as an example, we analysis its stress distribution and stiffness, getting its rule of stress distribution at the different loads, which can provide a theoretical basic for the design and optimization of the diaphragm.

For the characteristics of Diaphragm coupling, many scholars at home and abroad made a wide range of research. The research contents mainly involves coupling analysis of static and dynamic, which include the stress distribution under different load, the modal analysis and the analysis of the life [2] etc. From that, they established the formulas of stress and stiffness.

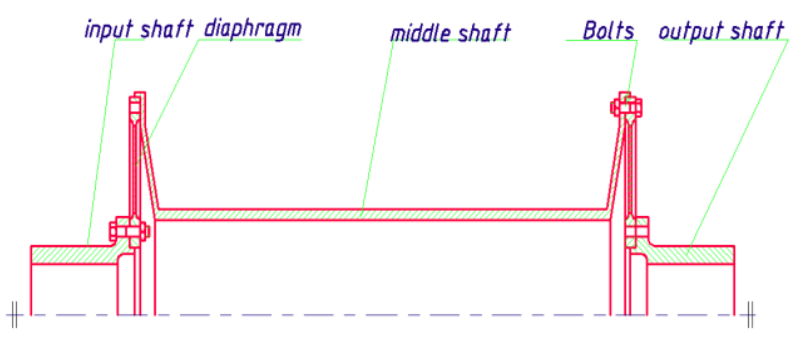

Figure 1. Diaphragm coupling

\section{The finite element model}

The common thickness calculation formula of diaphragm profile is described below:

$$
T=C / R^{\mathrm{n}}
$$

The profile of diaphragm is corresponding to linear, conical and hyperbola diaphragm $[3,4]$ when the value of the index is $0,1,2$ severally. This paper takes the conical diaphragm as research object. In order to facilitate analysis, the model was simplified, which we apply the APDL (ANSYS parametric design language) to create parametric model in ANSYS. Besides, taking the calculation accuracy in consideration, we apply the element type of Solid185 unit to mesh the model and refine the grid in transitional region where the hexahedral element is used as shown in figure 2,3 . 


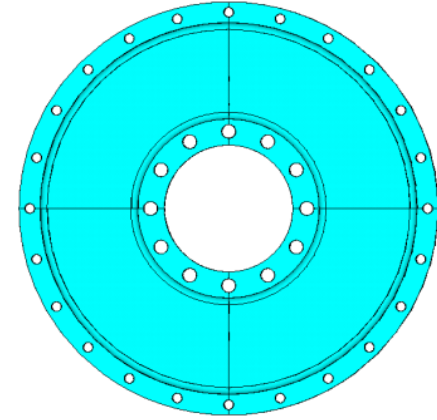

Figure 2. Geometry model of conical diaphragm

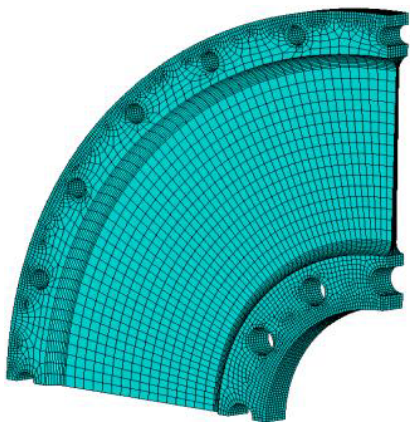

Figure 3. Local grid of conical diaphragm

\section{Stress analysis of the diaphragm}

\subsection{The stress value distribution of diaphragm under the action of torque}

Diaphragm suffers the torque that its value is $7958 \mathrm{Nm}$ when it works. Besides, the torque is equally distributed to the hole surface of the bolt after the torque are translated in to the pressure which according to cosine distribution. What's more, taking the action of torque in to consideration only, we fix the radial and axial displacement of the inner holes. Furthermore, all constraints are applied in outer holes. After solving, the stress contours of diaphragm and stress in profile along with the y axis distribution curve are shown.

As shown in figure 4,5, we can know that the value of maximum stress is $247 \mathrm{Mpa}$ when the Diaphragm suffer the torque only. The stress value in inner diameter of profile is about $239 \mathrm{Mpa}$. With the value of radius increase, the stress value decreases gradually and changes slowly.
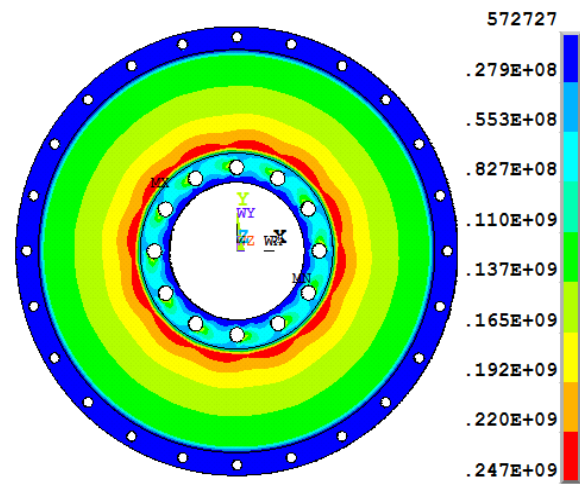

Figure 4. The stress caused by torque

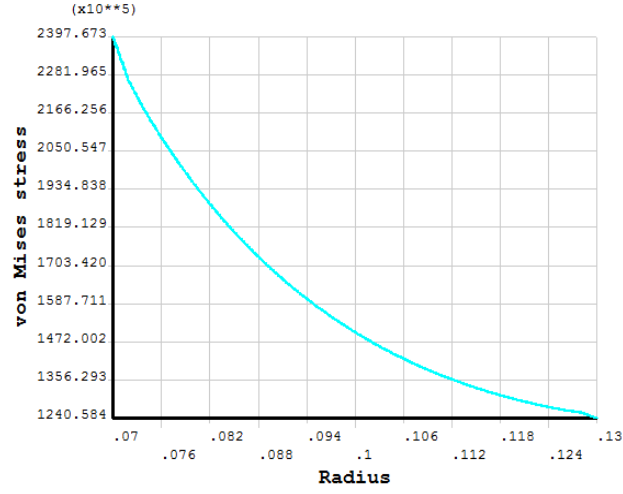

Figure 5. The stress distribution curve of the profile

\subsection{Stress value of diaphragm under the action of Centrifugal force}

We can know that the rotate speed of Diaphragm is 7200 rpm when Diaphragm works. Besides, we apply the load in the form of angular velocity, meanwhile we fix the angular and axial displacement of the all holes [5]. After solving, we can get the stress contours of diaphragm and stress of profile along with the y axis distribution curve.

As shown in the figure 6,7 , we can know that the value of maximum stress is $41.1 \mathrm{Mpa}$, and the stress value in outer diameter of profile is about $30.73 \mathrm{Mpa}$. With the value of the radius increase, the stress showed a trend that decreases firstly and then increase. Compared with the stress of torque load, it has smaller impact to the stress of the diaphragm.

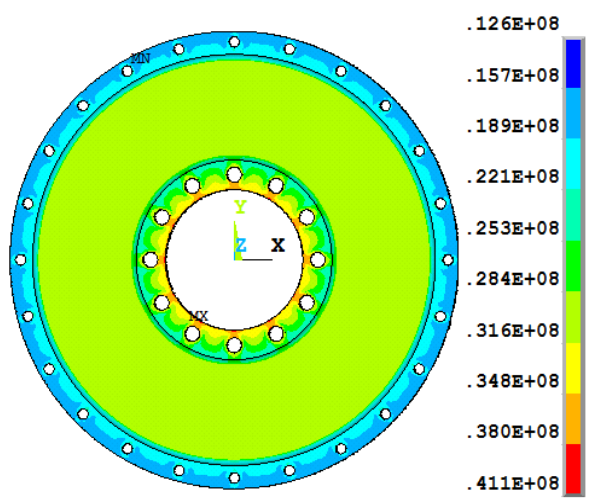

Figure 6. The stress caused by centrifugal force

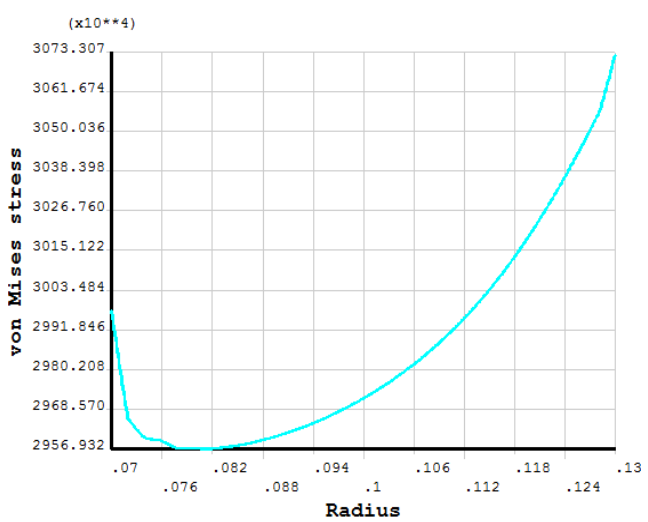

Figure 7. The stress distribution curve of the profile 


\subsection{Stress value of diaphragm under the action of Axial Deviation}

We can know that the axial deviation value of Diaphragm is $3 \mathrm{~mm}$ which is applied in inner bolt holes when Diaphragm works. In the process of analysis, we fix all constraints of the all outer holes ${ }^{[5]}$. After solving, we can get the stress contours of diaphragm and stress of profile along with the $y$ axis distribution curve.

As shown in the figure 8,9, we can know that the value of the maximum stress is $515 \mathrm{Mpa}$ when diaphragm suffer axial deviation only. Besides, the stress in inner and outer diameter of profile is about $381 \mathrm{Mpa}$. With the value of the radius increase, the value of stress displays a trend that decreases firstly and then increase. What's more, the stress value in inner and outer radius is almost equal. Compared with the load in the front, the axial deviation has greater impact to the stress value of the diaphragm.

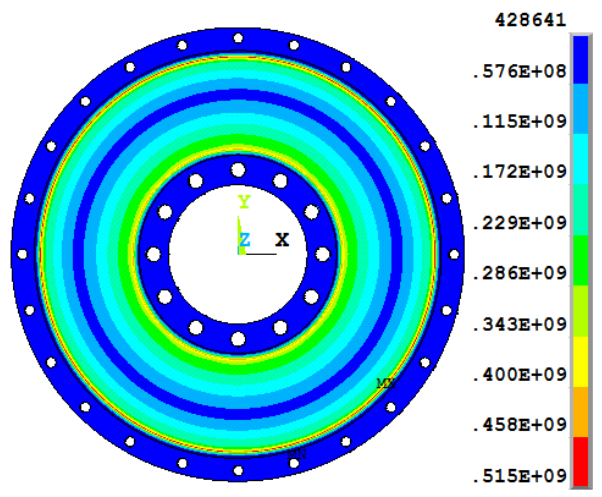

Figure 8. The stress caused by axial deviation

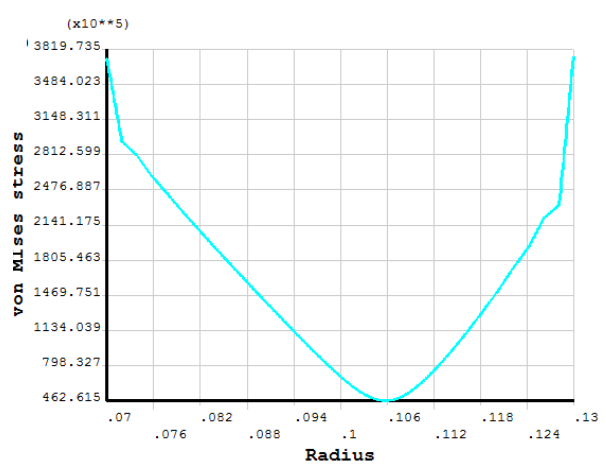

Figure 9. The stress distribution curve of the profile

\subsection{Stress value of diaphragm under the action of Angular Deviation}

Suppose that the diaphragm withstand value of angular deviation is $0.3^{\circ}$. We transform it to the axial displacement constraint which is applied in the inner bolt holes. All constraints of the outer bolt holes are fixed. After solving, the stress contours of diaphragm and stress of the profile along with the y axis distribution curve are shown

As shown in the figure 10,11, we can know that the value of maximum stress is $82 \mathrm{Mpa}$ when the diaphragm suffer angular deviation only. The stress value in inner diameter of working profile is about $72.23 \mathrm{Mpa}$. With the value of the radius increase, the stress value shows a trend that decreases firstly and then increase.

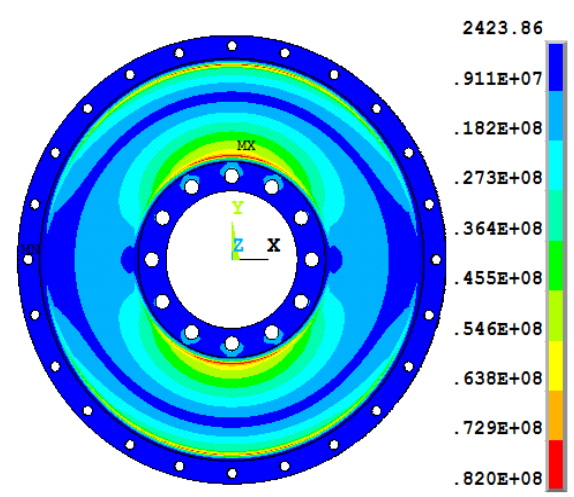

Figure 10. The stress caused by angular deviation

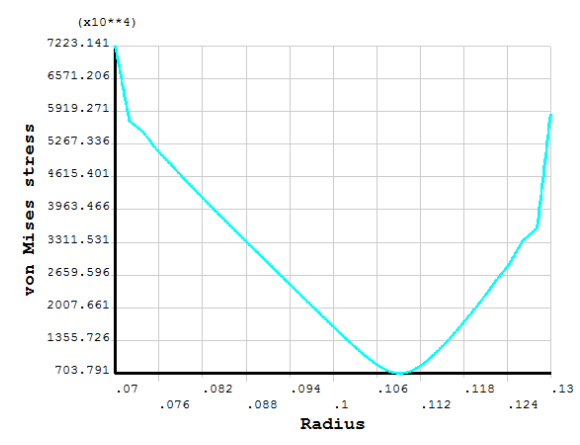

Figure 11. The stress distribution curve of the profile

\subsection{Stress value of diaphragm under the action of variety loads}

We apply four kinds of aforementioned loads at the same time. By fixing the radial and axial displacement constraint of the outer holes, we can get the stress contours of diaphragm and stress value in working profile along with the $\mathrm{Y}$ axis distribution curve after solving.

As shown in the figure 10,11, we can know that the value of the maximum stress is $598 \mathrm{Mpa}$ when diaphragm suffer multiple loads. The stress value in inner diameter of working profile is about $510 \mathrm{Mpa}$. With the radius value increase, the stress value showed a trend that decreases firstly and then increase. The distribution form of stress value is the same as the stress caused by axial deviation.

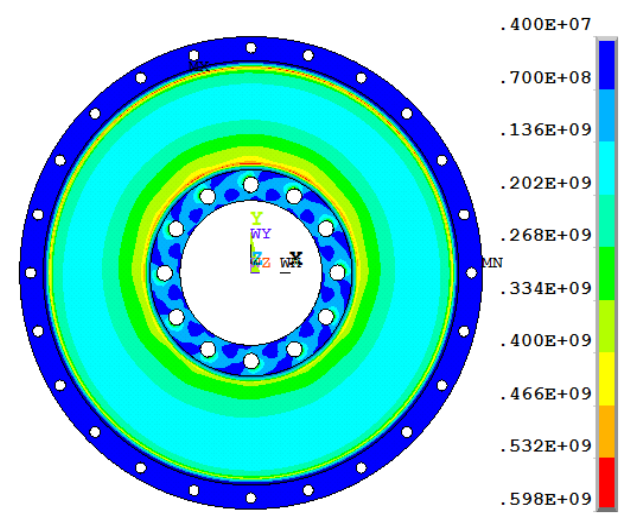

Figure 12. The stress caused by variety loads 


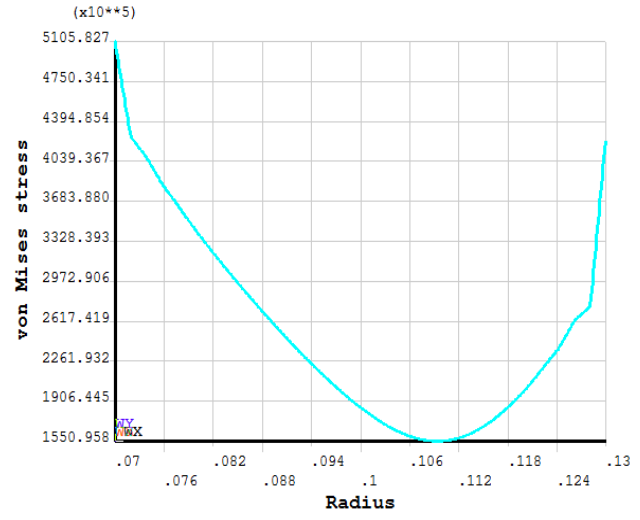

Figure 13. The stress distribution curve of the profile Based on above, the max stress in profile and its takes place is shown in Table 1 .

Table 1. The max stress in profile and its takes place

\begin{tabular}{|l|c|c|c|c|c|}
\hline & torque & $\begin{array}{c}\text { Centrifugal } \\
\text { force }\end{array}$ & $\begin{array}{c}\text { Axial } \\
\text { Deviation }\end{array}$ & $\begin{array}{c}\text { Angular } \\
\text { Deviation }\end{array}$ & $\begin{array}{c}\text { variety } \\
\text { loads }\end{array}$ \\
\hline Stress & 247 & 41.1 & 515 & 82 & 598 \\
\hline place & inside & outside & outside & inside & outside \\
\hline
\end{tabular}

\section{Stiffness analysis of diaphragm}

Diaphragm will produce the torsional stiffness when suffer the load of torque, and axial stiffness when suffer axial deviation. Besides, Diaphragm will produce angular stiffness $[6,7]$ when suffer the angular deviation. In this section, we will study on the change of the stiffness when Diaphragm suffers different loads. From that, we can get three kinds of stiffness curve under the different loads are showed in fig 14 to fig 16.

As shown in the figures, we can know that the deflection angle of diaphragm increase linearly and the slope of curve is torsional stiffness, its value is about 7.04 $\times 10^{7} \mathrm{Nm} / \mathrm{Rad}$ when the diaphragm suffers the different torque load. Besides, the axial reaction force increases linearly and the value of axial stiffness is about $1.27 \times$ $10^{6} \mathrm{~N} / \mathrm{m}$ when the diaphragm suffers the load of axial deviation [7]. What's more, the torque reaction increases linearly and the value of angular stiffness is about $7435.9 \mathrm{Nm} / \mathrm{Rad}$ when the diaphragm suffers load of different angular deviation.

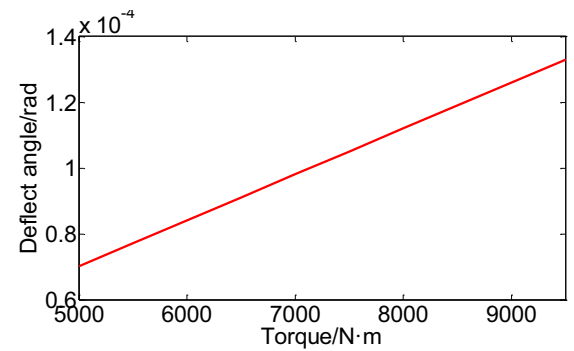

Figure 14. The torsional angle curve of diaphragm

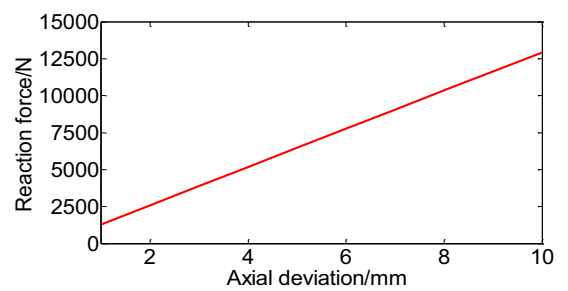

Figure 15. The axial reaction force curve of diaphragm

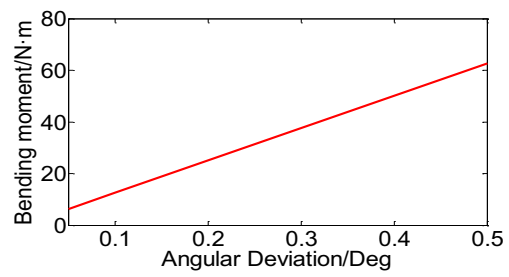

Figure 16. The bending moment curve of diaphragm

\section{Conclusion}

In this paper, we study on the distribution of the stress and three kinds of stiffness for conical diaphragm when the diaphragm suffers different loads. From that, the results show that the value of maximum stress usually appears in the outer or inner transition region. Compared with the other load, the axial deviation has a greater influence to the distribution of the stress which generates the greatest stress. But the diaphragm coupling usually bear the bigger stress, due to that it usually suffers the several loads that has mentioned above. In the situation that the structure parameters of the diaphragm is constant, the three kinds of stiffness for conical diaphragm is constant when the diaphragm suffer different loads.

\section{References}

1. H. Liao, Y.L.He, J. Du, Strength and Fatigue Finite Element Analysis of Diaphragm in Diaphragm Coupling, Machinery \& Electronics, 05,5(2008)

2. Z.G.Qiu, F.H.Zhang, J.H.bai, the design and FEM analysis of flexible diaphragm coupling' web curve, Machinery Design\&Manufacture, 7, 2(2010)

3. Y. Peng, G.L.Deng, L.F.Xing, Diaphragm strength calculation method, Ship Science and Technology, 35,5(2003)

4. Y. Peng, Y.Zhao, X.X.Liu, Analysis of different type mask plate characteristic, Ship Science and Technology,35,5(2013)

5. S. Liu, X.F.Wang, Analysis of axial misalignment based on ANSYS of flexible couplings, Mechanical Research and Application, 16,2(2003)

6. L.F.Shentu,X.Y.Zhang, Analysis Axial displacement compensation capacity of Joint Flexible Laminated Membrane Coupling (JFLMC), 19,2(2003)

7. W.P.Chen, Y.M.Ma, analysis axis stiffness of diaphragm coupling, ship engineering, 32,4(2010) 